\title{
Contractible edges in 2-connected locally finite graphs
}

\author{
Tsz Lung Chan \\ Mathematisches Seminar \\ Universität Hamburg \\ Hamburg, Germany \\ tlchantl@gmail.com
}

Submitted: May 30, 2014; Accepted: May 25, 2015; Published: Jun 15, 2015

Mathematics Subject Classifications: 05C40, 05C45, 05C63

\begin{abstract}
In this paper, we prove that every contraction-critical 2-connected infinite graph has no vertex of finite degree and contains uncountably many ends. Then, by investigating the distribution of contractible edges in a 2-connected locally finite infinite graph $G$, we show that the closure of the subgraph induced by all the contractible edges in the Freudenthal compactification of $G$ is 2-arc-connected. Finally, we characterize all 2-connected locally finite outerplanar graphs nonisomorphic to $K_{3}$ as precisely those graphs such that every vertex is incident to exactly two contractible edges as well as those graphs such that every finite bond contains exactly two contractible edges.
\end{abstract}

Keywords: contractible edge, Hamilton cycle, outerplanar, infinite graph

\section{Introduction}

Since the pioneering work of Tutte [11] who proved that every 3-connected finite graph nonisomorphic to $K_{4}$ contains a contractible edge, a lot of research has been done on contractible edges in finite graphs. One may consult the survey paper by Kriesell [8] for details.

For any 2-connected graph nonisomorphic to $K_{3}$, we have the well-known fact that every edge can either be deleted or contracted so that the resulting graph remains 2connected. This immediately leads to the following result.

Theorem 1. Let $G$ be a 2-connected finite graph nonisomorphic to $K_{3}$. Then the subgraph induced by all the contractible edges in $G$ is 2-connected. 
$\mathrm{Wu}[12]$ investigated the distribution of contractible elements in matroids and extended Theorem 1 to simple 2-connected matroids. He also characterized all simple 2-connected matroids $M$ having exactly $r(M)+1$ contractible elements (where $r(M)$ is the rank of $M$ ) as those matroids isomorphic to a graphic matroid of an outerplanar Hamiltonian graph.

Theorem 2 (Wu [12]). Let $G$ be a 2-connected finite graph nonisomorphic to $K_{3}$. Then every vertex of $G$ is incident to exactly two contractible edges if and only if $G$ is outerplanar.

On the other hand, only a few results were known for contractible edges in infinite graphs. For example, Mader [10] showed that every contraction-critical locally finite infinite graph has infinitely many triangles. Kriesell [9] provided a method of constructing contraction-critical $k$-connected infinite graphs $(k \geqslant 2)$. In Section 3 , we will prove that every contraction-critical 2-connected infinite graph contains vertices of infinite degree only and has uncountably many ends.

A natural way to extend Theorems 1 and 2 is to consider locally finite infinite graphs. Notice that Theorem 1 is no longer true as demonstrated by the infinite double ladder (the cartesian product of a double ray and $K_{2}$ ). The subgraph $G_{C}$ induced by all the contractible edges is the disjoint union of two double rays and is not even connected. Interestingly, the situation changes dramatically by looking at the graph from a topological viewpoint as introduced by Diestel and Kühn $[4,5,6]$. By adding the two ends of the double ladder to $G_{C}$, the resulting closure $\overline{G_{C}}$ is a circle and is 2-arc-connected. In Section 4 , we will prove that for every 2 -connected locally finite infinite graph $G, \overline{G_{C}}$ is 2-arc-connected.

Returning to Theorem 2, the backward direction is straightforward. For the forward direction, by Theorem $1, G_{C}$ is spanning and 2-connected. Since every vertex is incident to exactly two contractible edges, $G_{C}$ is a Hamilton cycle. Then it is easy to see that $G$ is outerplanar. When extending to locally finite infinite graphs, we now need the nontrivial statement that if $G$ is a 2-connected locally finite infinite graph such that every vertex is incident to exactly two contractible edges, then $\overline{G_{C}}$ is a Hamilton circle. This will be proved in Section 5. We will use it to prove an infinite analog of Theorem 2 for any 2 -connected locally finite graph $G$ nonisomorphic to $K_{3}$. Also we will show that $G$ is outerplanar if and only if every finite bond of $G$ contains exactly two contractible edges.

\section{Definitions}

All basic graph-theoretical terminology can be found in Diestel [3]. Unless otherwise stated, all graphs considered in this paper can be finite or infinite. An edge of a $k$ connected graph is said to be $k$-contractible if its contraction results in a $k$-connected graph. Otherwise, it is called $k$-non-contractible. A $k$-connected graph in which every edge is $k$-non-contractible is called contraction-critical $k$-connected. We simply write 2 contractible as contractible. Let $G=(V, E)$ be a 2-connected graph. Denote the set of all contractible edges in $G$ by $E_{C}$ and the subgraph induced by all the contractible edges by $G_{C}:=\left(V, E_{C}\right)$. Let $X$ and $Y$ be two disjoint subsets of $V$. An $X-Y$ path $P$ is a path such 
that only the starting vertex of $P$ lies in $X$ and only the ending vertex of $P$ lies in $Y$. Denote the set of all edges between $X$ and $Y$ by $E_{G}(X, Y)$. If $X$ and $Y$ form a partition of $V$, then $E_{G}(X, Y)$ is called a cut. A minimal non-empty cut is a bond. Denote the set of all edges incident to a vertex $x$ by $E_{G}(x)$ and the set of all neighbors of $x$ by $N_{G}(x)$. Define $N_{G}(X):=\left(\bigcup_{x \in X} N_{G}(x)\right) \backslash X$. A set $S$ of $k$ vertices is called an $k$-separator if $G-S$ is not connected.

Let $G$ be a locally finite graph. A ray is a 1-way infinite path, a double ray is a 2-way infinite path, and the subrays of a ray or double ray are its tails. An end is an equivalence class of rays where two rays are equivalent if no finite set of vertices separates them. Denote the set of the ends by $\Omega(G)$. We define a topological space, denoted by $|G|$, on $G$ together with its ends, which is known as the Freudenthal compactification of $G$ as follows. View $G$ as a 1-complex. Thus, every edge is homeomorphic to the unit interval. The basic open neighborhoods of a vertex $x$ consists of a choice of half-open half edges $[x z)$, one for each incident edge $x y$, where $z$ is any interior point of $x y$. For an end $\omega \in \Omega(G)$, we take as a basic open neighborhood the set of the form: $\hat{C}(S, \omega):=C(S, \omega) \cup \Omega(S, \omega) \cup \stackrel{\circ}{E}(S, \omega)$, where $S \subseteq V$ is a finite set of vertices, $C(S, \omega)$ is the component of $G-S$ in which every ray from $\omega$ has a tail, $\Omega(S, \omega)$ is the set of all ends whose rays have a tail in $C(S, \omega)$, and $\stackrel{\circ}{E}(S, \omega)$ is the set of all interior points of edges between $S$ and $C(S, \omega)$. Let $H$ be a subgraph of $G$. Then the closure of $H$ in $|G|$ is called a standard subspace and is denoted by $\bar{H}$. We say $H$ contains a point $x$ of $|G|$ if $x \in \bar{H}$.

Let $X$ and $Y$ be two topological spaces. A continuous map from the unit interval $[0,1]$ to $X$ is a path in $X$. A homeomorphic image of $[0,1]$ in $X$ is called an $\operatorname{arc}$ in $X$. This induces an ordering $<$ for the points in the arc. The images of 0 and 1 are the endpoints of the arc. An arc in $X$ with endpoints $x$ and $y$ is called an $x-y$ arc. A homeomorphic image of the unit circle in $X$ is called a circle in $X$. A (path-) component of $X$ is a maximal (path-)connected set in $X . X$ is 2-connected (2-arc-connected) if for all $x \in X, X \backslash x$ is connected (arc-connected). We say $X$ can be embedded in $Y$ if there exists an injective continuous function $\phi: X \rightarrow Y$ such that $X$ is homeomorphic to $\phi(X)$ in the subspace topology of $Y$. Then $\phi$ is called an embedding of $X$ in $Y$. Take $Y$ to be $\mathbb{R}^{2}$. A component of $\mathbb{R}^{2} \backslash \phi(X)$ is called a face of $\phi(X)$ in $\mathbb{R}^{2}$. A graph $G$ is planar if $G$ can be embedded in $\mathbb{R}^{2}$. A graph $G$ is outerplanar if there exists an embedding $\phi$ of $G$ in $\mathbb{R}^{2}$ such that there is a face $f$ of $\phi(G)$ in $\mathbb{R}^{2}$ whose boundary $\partial f$ contains all the vertices of $G$. Chartrand and Harary [2] characterized outerplanar finite graphs as precisely those graphs that do not contain a $K_{2,3^{-}}$or $K_{4^{-}}$subdivision.

Suppose $A$ is an arc in $|G|$ and $x$ is a vertex in $A$. Then the vertex immediately before $x$ in $A$ if exists is denoted by $x^{-}$and the vertex immediately after $x$ in $A$ if exists is denoted by $x^{+}$. An arc in $|G|$ is an $\omega$-arc if the end $\omega$ is one of its endpoints and unless otherwise stated, it corresponds to the image of 1 . Following Bruhn and Stein [1], we define the end degree of an end $\omega$ in $G$ as the supremum over the cardinalities of sets of edge-disjoint rays in $\omega$, and denote this number by $\operatorname{deg}_{G}(\omega)$. In fact, they proved that this is equal to the supremum over the cardinalities of sets of edge-disjoint $\omega$-arcs in $|G|$. For a subgraph $H$ of $G$, define the degree of $\omega$ in $H$ as the supremum over the cardinalities of sets of edge-disjoint $\omega$-arcs in $\bar{H}$ which is denoted by $\operatorname{deg}_{H}(\omega)$. 


\section{Contraction-critical 2-connected infinite graphs}

It is well-known that the only contraction-critical 2 -connected finite graph is $K_{3}$. However, there are infinitely many contraction-critical 2-connected infinite graphs as shown by the following construction due to Kriesell [9]. Define $G_{0}:=\emptyset$ and let $G_{1}$ be any 2-connected finite graph. Suppose we have constructed $G_{n}$ such that $G_{n-1} \subsetneq G_{n}$. For each edge $x y$ in $E\left(G_{n}\right) \backslash E\left(G_{n-1}\right)$, add a new $x$-y path of length at least 2 . The resulting graph is $G_{n+1}$. Repeat the process inductively. Then the graph $G:=\bigcup_{i \geqslant 1} G_{i}$ is a contractioncritical 2-connected infinite graph. Note that $G$ has no vertex of finite degree and has uncountably many ends. We will show that this holds in general for any contractioncritical 2-connected infinite graph. First, we state a fundamental fact about contractible edges in 2-connected graphs.

Lemma 3. Let $G$ be a 2-connected graph nonisomorphic to $K_{3}$ and e be an edge of $G$. Then $G-e$ or $G / e$ is 2-connected.

Now, we can develop some tools that will be used for the rest of the paper.

Lemma 4. Let $G$ be a 2-connected graph nonisomorphic to $K_{3}$, and $e$ and $f$ be two non-contractible edges of $G$. Then $f$ is a non-contractible edge of $G-e$.

Proof. By Lemma 3, $G-e$ is 2-connected. Since $V(f)$ is a 2-separator of $G, V(f)$ is also a 2-separator of $G-e$ and $f$ is a non-contractible edge of $G-e$.

Lemma 5. Let $G$ be a 2-connected graph nonisomorphic to $K_{3}$ and $F$ be a finite subset of $E(G)$.

(a) If $G-F$ is disconnected, then $F$ contains at least two contractible edges.

(b) If $G-F$ is connected but not 2-connected, then F contains at least one contractible edge.

Proof. For (a), suppose $F$ contains at most one contractible edge. Then by Lemma 3 and 4 , we can delete all the non-contractible edges in $F$ and the resulting graph is still 2-connected, a contradiction.

For (b), suppose all edges in $F$ are non-contractible. Then by Lemma 3 and 4 , we can delete all edges in $F$ and $G-F$ is still 2-connected, a contradiction.

Lemma 6. Let $G$ be a 2-connected graph nonisomorphic to $K_{3}$. Let $\{x, y\}$ be a 2-separator of $G$ and $C$ be a component of $G-x-y$. If $\left|E_{G}(x, C)\right|$ is finite, then $E_{G}(x, C)$ contains a contractible edge.

Proof. Note that $y$ is a cutvertex of $G-E_{G}(x, C)$. By Lemma $5(\mathrm{~b}), E_{G}(x, C)$ contains a contractible edge.

Lemma 7. Let $G$ be a 2-connected graph nonisomorphic to $K_{3}$ and $x$ be a vertex of $G$. Suppose all edges incident to $x$ are non-contractible. Then 
(a) $x$ has infinite degree.

(b) For any edge $x y$ incident to $x$, every component of $G-x-y$ contains infinitely many neighbors of $x$.

Proof. For (a), Suppose $x$ has finite degree. By applying Lemma $5\left(\right.$ a) to $E_{G}(x), x$ is incident to at least two contractible edges, a contradiction.

For (b), let $C$ be a component of $G-x-y$. By Lemma $6, E_{G}(x, C)$ contains infinitely many edges.

Theorem 8. Let $G$ be a contraction-critical 2-connected infinite graph. Then every vertex of $G$ has infinite degree and $G$ has uncountably many ends.

Proof. By Lemma 7(a), every vertex of $G$ has infinite degree.

Next, we will construct a rooted binary infinite tree $T$ in $G$ together with edges incident to each vertex of $T$ with the following properties:

(1) The root of $T$ is denoted by $x$.

(2) The vertices of $T$ are denoted by $x_{n_{1} n_{2} \ldots n_{k}}$ where $k \in \mathbb{N}$ and $n_{i} \in\{0,1\}$ for $1 \leqslant i \leqslant k$. For $k=0$, define $x_{n_{1} n_{2} \ldots n_{k}}:=x$.

(3) Each vertex $x_{n_{1} n_{2} \ldots n_{k}}$ of $T$ is adjacent to two vertices $x_{n_{1} n_{2} \ldots n_{k} 0}$ and $x_{n_{1} n_{2} \ldots n_{k} 1}$ in $T$.

(4) For each vertex $x_{n_{1} n_{2} \ldots n_{k}}$ of $T$, there exists an edge $x_{n_{1} n_{2} \ldots n_{k}} y_{n_{1} n_{2} \ldots n_{k}}$ in $G$ such that $y_{n_{1} n_{2} \ldots n_{k}}$ does not lie in $T$.

(5) The subtree of $T$ rooted at $x_{n_{1} n_{2} \ldots n_{k}}$ is defined as

$T_{n_{1} n_{2} \ldots n_{k}}:=T\left[\bigcup_{i=0}^{\infty} \bigcup_{\left(m_{1}, m_{2}, \ldots, m_{i}\right) \in\{0,1\}^{i}} x_{n_{1} n_{2} \ldots n_{k} m_{1} m_{2} \ldots m_{i}}\right]$.

For fixed $n_{1}, n_{2}, \ldots, n_{k}, \bigcup_{j=0}^{k}\left\{x_{n_{1} n_{2} \ldots n_{j}}, y_{n_{1} n_{2} \ldots n_{j}}\right\}$ separates $T_{n_{1} n_{2} \ldots n_{k} 0}$ and $T_{n_{1} n_{2} \ldots n_{k} 1}$ in $G$.

Each ray in $T$ starting at $x$ is of the form: $x x_{n_{1}} x_{n_{1} n_{2}} x_{n_{1} n_{2} n_{3}} \ldots$ Let $R:=x x_{n_{1}} x_{n_{1} n_{2}}$ $x_{n_{1} n_{2} n_{3}} \ldots$ and $Q:=x x_{m_{1}} x_{m_{1} m_{2}} x_{m_{1} m_{2} m_{3}} \ldots$ be two distinct rays in $T$. Then there exists a smallest $k$ such that $n_{i}=m_{i}$ for all $i \leqslant k$ and $n_{k+1} \neq m_{k+1}$. By property (5) above, $\bigcup_{j=0}^{k}\left\{x_{n_{1} n_{2} \ldots n_{j}}, y_{n_{1} n_{2} \ldots n_{j}}\right\}$ separates $R$ and $Q$ in $G$. Therefore, each ray in $T$ starting at $x$ belongs to a unique end of $G$, and $G$ has uncountably many ends.

Now, it remains to construct $T$ inductively. Let $x$ be any vertex in $G$. Define $T_{0}:=(\{x\}, \emptyset)$. Choose any edge incident to $x$ in $G$, say $x y$. Let $C_{0}$ and $C_{1}$ be any two components of $G-x-y$. Let $x_{0}$ be a neighbor of $x$ in $C_{0}$ and $x_{1}$ be a neighbor of $x$ in $C_{1}$. Define $T_{1}:=\left(\left\{x, x_{0}, x_{1}\right\},\left\{x x_{0}, x x_{1}\right\}\right)$. Note that $N_{G}\left(C_{0}\right) \subseteq\{x, y\}$ and $N_{G}\left(C_{1}\right) \subseteq\{x, y\}$. Also, $G-C_{0}$ and $G-C_{1}$ are both connected.

Suppose we have constructed the rooted binary tree $T_{k}$ where

$$
\begin{aligned}
& V\left(T_{k}\right):=\bigcup_{i=0}^{k} \bigcup_{\left(n_{1}, n_{2}, \ldots, n_{i}\right) \in\{0,1\}^{i}} x_{n_{1} n_{2} \ldots n_{i}} \text { and } \\
& E\left(T_{k}\right):=\bigcup_{i=0}^{k-1} \bigcup_{\left(n_{1}, n_{2}, \ldots, n_{i}\right) \in\{0,1\}^{i}}\left\{x_{n_{1} n_{2} \ldots n_{i}} x_{n_{1} n_{2} \ldots n_{i} 0}, x_{n_{1} n_{2} \ldots n_{i}} x_{n_{1} n_{2} \ldots n_{i} 1}\right\} \text { such that }
\end{aligned}
$$


(i) each vertex $x_{n_{1} n_{2} \ldots n_{i}}(0 \leqslant i \leqslant k)$ lies in a connected subgraph $C_{n_{1} n_{2} \ldots n_{i}}$ of $G$ (for $i=0, x_{n_{1} n_{2} \ldots n_{i}}:=x, y_{n_{1} n_{2} \ldots n_{i}}:=y$ and $\left.C_{n_{1} n_{2} \ldots n_{i}}:=G\right)$,

(ii) for each vertex $x_{n_{1} n_{2} \ldots n_{i}}(0 \leqslant i<k)$, we have found an edge $x_{n_{1} n_{2} \ldots n_{i}} y_{n_{1} n_{2} \ldots n_{i}}$ that lies in $C_{n_{1} n_{2} \ldots n_{i}}$ such that $C_{n_{1} n_{2} \ldots n_{i} 0}$ and $C_{n_{1} n_{2} \ldots n_{i} 1}$ are two components of $C_{n_{1} n_{2} \ldots n_{i}}-$ $x_{n_{1} n_{2} \ldots n_{i}}-y_{n_{1} n_{2} \ldots n_{i}}$ that are adjacent to $x_{n_{1} n_{2} \ldots n_{i}}$,

(iii) for fixed $n_{1}, n_{2}, \ldots, n_{i}(1 \leqslant i \leqslant k), N_{G}\left(C_{n_{1} n_{2} \ldots n_{i}}\right) \subseteq \bigcup_{j=0}^{i-1}\left\{x_{n_{1} n_{2} \ldots n_{j}}, y_{n_{1} n_{2} \ldots n_{j}}\right\}$,

(iv) for fixed $n_{1}, n_{2}, \ldots, n_{i}(1 \leqslant i \leqslant k), G-C_{n_{1} n_{2} \ldots n_{i}}$ is connected.

Now, for each vertex $x_{n_{1} n_{2} \ldots n_{k}}$ in $T_{k}$, since it has infinite degree and $N_{G}\left(x_{n_{1} n_{2} \ldots n_{k}}\right) \backslash$ $C_{n_{1} n_{2} \ldots n_{k}} \subseteq N_{G}\left(C_{n_{1} n_{2} \ldots n_{k}}\right)$ is finite by (iii), all but finitely many neighbors of $x_{n_{1} n_{2} \ldots n_{k}}$ lie in $C_{n_{1} n_{2} \ldots n_{k}}$. Let $z$ be a neighbor of $x_{n_{1} n_{2} \ldots n_{k}}$ in $C_{n_{1} n_{2} \ldots n_{k}}$ and $B:=C_{n_{1} n_{2} \ldots n_{k}}-x_{n_{1} n_{2} \ldots n_{k}}-z$. Suppose $B$ is connected. Since $B^{\prime}:=G-C_{n_{1} n_{2} \ldots n_{k}}$ is connected by (iv), $B$ and $B^{\prime}$ are the only two components of $G-x_{n_{1} n_{2} \ldots n_{k}}-z$. By Lemma $7(\mathrm{~b}), B^{\prime}$ contains infinitely many neighbors of $x_{n_{1} n_{2} \ldots n_{k}}$ contradicting $N_{G}\left(x_{n_{1} n_{2} \ldots n_{k}}\right) \backslash C_{n_{1} n_{2} \ldots n_{k}} \subseteq N_{G}\left(C_{n_{1} n_{2} \ldots n_{k}}\right)$ which is finite by (iii). Therefore, $B$ is not connected.

Note that at least one component of $B$ is adjacent to $x_{n_{1} n_{2} \ldots n_{k}}$. If not, then, by the 2connectedness of $G$, each component of $B$ has a neighbor in $N_{G}\left(C_{n_{1} n_{2} \ldots n_{k}}\right) \subseteq G-C_{n_{1} n_{2} \ldots n_{k}}$. By (iv), this implies $G-x_{n_{1} n_{2} \ldots n_{k}}-z$ is connected, a contradiction. Suppose there are two components of $B$, say $D$ and $D^{\prime}$, that are both adjacent to $x_{n_{1} n_{2} \ldots n_{k}}$. Then choose $y_{n_{1} n_{2} \ldots n_{k}}:=z, C_{n_{1} n_{2} \ldots n_{k} 0}:=D$ and $C_{n_{1} n_{2} \ldots n_{k} 1}:=D^{\prime}$. Suppose only one component of $B$ is adjacent to $x_{n_{1} n_{2} \ldots n_{k}}$, say $C$. Each component of $B$ other than $C$ is adjacent to $z$ by the connectedness of $C_{n_{1} n_{2} \ldots n_{k}}$ and has a neighbor in $N_{G}\left(C_{n_{1} n_{2} \ldots n_{k}}\right) \subseteq G-C_{n_{1} n_{2} \ldots n_{k}}$ by the 2-connectedness of $G$. Denote the union of components of $B$ other than $C$ by $C^{\prime}$. Since $G-C_{n_{1} n_{2} \ldots n_{k}}$ is connected by (iv), $C^{\prime \prime}:=G\left[\left(G-C_{n_{1} n_{2} \ldots n_{k}}\right) \cup C^{\prime}\right]$ is connected. Hence, $C$ and $C^{\prime \prime}$ are the only two components of $G-x_{n_{1} n_{2} \ldots n_{k}}-z$ and $N_{G}(C)=\left\{x_{n_{1} n_{2} \ldots n_{k}}, z\right\}$. Let $z^{\prime}$ be a neighbor of $x_{n_{1} n_{2} \ldots n_{k}}$ in $C$. Then one component $D$ of $C_{n_{1} n_{2} \ldots n_{k}}-x_{n_{1} n_{2} \ldots n_{k}}-z^{\prime}$ contains $z$ and $C^{\prime}$. Since $G-x_{n_{1} n_{2} \ldots n_{k}}-z^{\prime}$ is not connected, $D$ cannot be the only component of $C_{n_{1} n_{2} \ldots n_{k}}-x_{n_{1} n_{2} \ldots n_{k}}-z^{\prime}$. Let $D^{\prime}$ be any component of $C_{n_{1} n_{2} \ldots n_{k}}-x_{n_{1} n_{2} \ldots n_{k}}-z^{\prime}$ other than $D$. Then $D^{\prime}$ lies in $C$ and $N_{G}\left(D^{\prime}\right) \subseteq\left\{x_{n_{1} n_{2} \ldots n_{k}}, z^{\prime}\right\} \cup\left(N_{G}(C)-z\right)=\left\{x_{n_{1} n_{2} \ldots n_{k}}, z^{\prime}\right\}$. Now, choose $y_{n_{1} n_{2} \ldots n_{k}}:=z^{\prime}, C_{n_{1} n_{2} \ldots n_{k} 0}:=D$ and $C_{n_{1} n_{2} \ldots n_{k} 1}:=D^{\prime}$.

In both cases, $x_{n_{1} n_{2} \ldots n_{k}} y_{n_{1} n_{2} \ldots n_{k}}$ lies in $C_{n_{1} n_{2} \ldots n_{k}}$, and $C_{n_{1} n_{2} \ldots n_{k} 0}$ and $C_{n_{1} n_{2} \ldots n_{k} 1}$ are two components of $C_{n_{1} n_{2} \ldots n_{k}}-x_{n_{1} n_{2} \ldots n_{k}}-y_{n_{1} n_{2} \ldots n_{k}}$ that are adjacent to $x_{n_{1} n_{2} \ldots n_{k}}$. Let $x_{n_{1} n_{2} \ldots n_{k} 0}$ be a neighbor of $x_{n_{1} n_{2} \ldots n_{k}}$ in $C_{n_{1} n_{2} \ldots n_{k} 0}$ and $x_{n_{1} n_{2} \ldots n_{k} 1}$ be a neighbor of $x_{n_{1} n_{2} \ldots n_{k}}$ in $C_{n_{1} n_{2} \ldots n_{k} 1}$. Since $C_{n_{1} n_{2} \ldots n_{k} 0} \subseteq C_{n_{1} n_{2} \ldots n_{k}}$ and $C_{n_{1} n_{2} \ldots n_{k} 1} \subseteq C_{n_{1} n_{2} \ldots n_{k}}, N_{G}\left(C_{n_{1} n_{2} \ldots n_{k} 0}\right) \subseteq$ $N_{G}\left(C_{n_{1} n_{2} \ldots n_{k}}\right) \cup\left\{x_{n_{1} n_{2} \ldots n_{k}}, y_{n_{1} n_{2} \ldots n_{k}}\right\} \subseteq \bigcup_{j=0}^{k}\left\{x_{n_{1} n_{2} \ldots n_{j}}, y_{n_{1} n_{2} \ldots n_{j}}\right\}$ and $N_{G}\left(C_{n_{1} n_{2} \ldots n_{k} 1}\right) \subseteq$ $N_{G}\left(C_{n_{1} n_{2} \ldots n_{k}}\right) \cup\left\{x_{n_{1} n_{2} \ldots n_{k}}, y_{n_{1} n_{2} \ldots n_{k}}\right\} \subseteq \bigcup_{j=0}^{k}\left\{x_{n_{1} n_{2} \ldots n_{j}}, y_{n_{1} n_{2} \ldots n_{j}}\right\}$ by (iii). By the connectedness of $C_{n_{1} n_{2} \ldots n_{k}}$, every component of $C_{n_{1} n_{2} \ldots n_{k}}-x_{n_{1} n_{2} \ldots n_{k}}-y_{n_{1} n_{2} \ldots n_{k}}$ has a neighbor in $\left\{x_{n_{1} n_{2} \ldots n_{k}}, y_{n_{1} n_{2} \ldots n_{k}}\right\}$. For $n_{k+1} \in\{0,1\}$, denote the union of the components of $C_{n_{1} n_{2} \ldots n_{k}}-$ $x_{n_{1} n_{2} \ldots n_{k}}-y_{n_{1} n_{2} \ldots n_{k}}$ other than $C_{n_{1} n_{2} \ldots n_{k} n_{k+1}}$ by $U_{n_{k+1}}$. Then $G\left[x_{n_{1} n_{2} \ldots n_{k}} y_{n_{1} n_{2} \ldots n_{k}} \cup U_{n_{k+1}}\right]$ is connected. Since $x_{n_{1} n_{2} \ldots n_{k-1}} \in G-C_{n_{1} n_{2} \ldots n_{k}}, x_{n_{1} n_{2} \ldots n_{k-1}} x_{n_{1} n_{2} \ldots n_{k}} \in E(G)$ and $G-C_{n_{1} n_{2} \ldots n_{k}}$ is connected by (iv), $G-C_{n_{1} n_{2} \ldots n_{k} n_{k+1}}:=G\left[\left(G-C_{n_{1} n_{2} \ldots n_{k}}\right) \cup x_{n_{1} n_{2} \ldots n_{k}} y_{n_{1} n_{2} \ldots n_{k}} \cup U_{n_{k+1}}\right]$ is connected. 
Define $T_{k+1}$ where $V\left(T_{k+1}\right):=\bigcup_{i=0}^{k+1} \bigcup_{\left(n_{1}, n_{2}, \ldots, n_{i}\right) \in\{0,1\}^{i}} x_{n_{1} n_{2} \ldots n_{i}}$ and $E\left(T_{k+1}\right):=\bigcup_{i=0}^{k} \bigcup_{\left(n_{1}, n_{2}, \ldots, n_{i}\right) \in\{0,1\}^{i}}\left\{x_{n_{1} n_{2} \ldots n_{i}} x_{n_{1} n_{2} \ldots n_{i} 0}, x_{n_{1} n_{2} \ldots n_{i}} x_{n_{1} n_{2} \ldots n_{i} 1}\right\}$.

Finally, define $T:=\bigcup_{k=0}^{\infty} T_{k}$. It is easy to see that $T$ satisfies properties (1) through (4). Let $z_{0}$ and $z_{1}$ be any vertices in $T_{n_{1} n_{2} \ldots n_{k} 0}$ and $T_{n_{1} n_{2} \ldots n_{k} 1}$ respectively. Then $z_{0}$ is of the form $x_{n_{1} n_{2} \ldots n_{k} 0 p_{1} p_{2} \ldots p_{i}}$ while $z_{1}$ is of the form $x_{n_{1} n_{2} \ldots n_{k} 1 q_{1} q_{2} \ldots q_{j}}$. We have $z_{0}=x_{n_{1} n_{2} \ldots n_{k} 0 p_{1} p_{2} \ldots p_{i}} \in$ $C_{n_{1} n_{2} \ldots n_{k} 0 p_{1} p_{2} \ldots p_{i}} \subseteq C_{n_{1} n_{2} \ldots n_{k} 0 p_{1} p_{2} \ldots p_{i-1}} \subseteq \ldots \subseteq C_{n_{1} n_{2} \ldots n_{k} 0}$ and $z_{1}=x_{n_{1} n_{2} \ldots n_{k} 1 q_{1} q_{2} \ldots p_{j}} \in$ $C_{n_{1} n_{2} \ldots n_{k} 1 q_{1} q_{2} \ldots q_{j}} \subseteq C_{n_{1} n_{2} \ldots n_{k} 1 q_{1} q_{2} \ldots q_{j-1}} \subseteq \ldots \subseteq C_{n_{1} n_{2} \ldots n_{k} 1}$. Therefore, $T_{n_{1} n_{2} \ldots n_{k} 0} \subseteq C_{n_{1} n_{2} \ldots n_{k} 0}$ and $T_{n_{1} n_{2} \ldots n_{k} 1} \subseteq C_{n_{1} n_{2} \ldots n_{k} 1}$. Since $\bigcup_{j=0}^{k}\left\{x_{n_{1} n_{2} \ldots n_{j}}, y_{n_{1} n_{2} \ldots n_{j}}\right\}$ contains both $N_{G}\left(C_{n_{1} n_{2} \ldots n_{k} 0}\right)$ and $N_{G}\left(C_{n_{1} n_{2} \ldots n_{k} 1}\right)$, it separates $C_{n_{1} n_{2} \ldots n_{k} 0}$ and $C_{n_{1} n_{2} \ldots n_{k} 1}$ in $G$ and thus separates $T_{n_{1} n_{2} \ldots n_{k} 0}$ and $T_{n_{1} n_{2} \ldots n_{k} 1}$ in $G$. Hence, Property (5) holds for $T$ and the proof is complete.

\section{Subgraph induced by all the contractible edges}

In this section, we will extend Theorem 1 to any 2-connected locally finite infinite graph $G$ and prove that $\overline{G_{C}}$ is 2 -arc-connected. Note that Lemma 5 (a) implies that every vertex is incident to at least two contractible edges. Hence, $G_{C}$ is spanning. Using the following two lemmas, it is easy to see that $\overline{G_{C}}$ is arc-connected.

Lemma 9 (Diestel [3]). Let $G$ be a locally finite graph. Then a standard subspace of $|G|$ is connected if and only if it contains an edge from every finite cut of $G$ of which it meets both sides.

Lemma 10 (Diestel and Kühn [6]). If $G$ is a locally finite graph, then every closed connected subspace of $|G|$ is arc-connected.

Theorem 11. Let $G$ be a 2-connected locally finite infinite graph and $G_{C}$ be the subgraph induced by all the contractible edges in $G$. Then $\overline{G_{C}}$ is arc-connected.

Proof. Let $F$ be any finite cut of $G$. By Lemma 5(a), $F$ contains at least two edges in $G_{C}$. Hence, $\overline{G_{C}}$ is connected by Lemma 9 . By Lemma $10, \overline{G_{C}}$ is arc-connected.

Next, we prove that $\overline{G_{C}}$ is 2 -connected.

Lemma 12. Let $G$ be a 2-connected locally finite infinite graph and $x$ be a point of $|G|$. Suppose there is a partition $\left(X, X^{\prime}\right)$ of $V(G \backslash x)$ such that $E_{G}\left(X, X^{\prime}\right)$ is non-empty and all edges in $E_{G}\left(X, X^{\prime}\right)$ are non-contractible. Then $G$ contains a subdivision of a 1-way infinite ladder $L$ consisting of two disjoint rays: $R:=x_{0} P_{1} x_{1} P_{2} x_{2} \ldots$ and $R^{\prime}:=x_{0}^{\prime} P_{1}^{\prime} x_{1}^{\prime} P_{2}^{\prime} x_{2}^{\prime} \ldots$ with the rungs of the ladder being $x_{0} x_{0}^{\prime}, x_{1} x_{1}^{\prime}, x_{2} x_{2}^{\prime}, \ldots$, all of which are $X-X^{\prime}$ edges such that $x \notin \bar{L}$.

Proof. Since $G$ is 2-connected, $\left|E_{G}\left(X, X^{\prime}\right)\right| \geqslant 2$ unless $x$ is a vertex and $\left|E_{G}\left(X, X^{\prime}\right)\right|=1$. Consider any $X-X^{\prime}$ edge $x_{0} x_{0}^{\prime}$ that does not contain $x$. Let $C$ be the component of $G-x_{0}-x_{0}^{\prime}$ containing $x$ and $C_{1}$ be a component of $G-x_{0}-x_{0}^{\prime}$ not containing $x$.

Suppose we have constructed the finite ladder $L_{k}$ consisting of two disjoint paths $R_{k}:=x_{0} P_{1} x_{1} P_{2} x_{2} \ldots x_{k-1} P_{k} x_{k}$ and $R_{k}^{\prime}:=x_{0}^{\prime} P_{1}^{\prime} x_{1}^{\prime} P_{2}^{\prime} x_{2}^{\prime} \ldots x_{k-1}^{\prime} P_{k}^{\prime} x_{k}^{\prime}$ with the rungs of the 
ladder being $x_{0} x_{0}^{\prime}, x_{1} x_{1}^{\prime}, \ldots x_{k} x_{k}^{\prime}$, all of which are $X-X^{\prime}$ edges such that $L_{k} \subseteq G\left[C_{1} \cup x_{0} \cup x_{0}^{\prime}\right]$ and $G\left[C \cup L_{k}-x_{k}-x_{k}^{\prime}\right]$ is connected. Let $C_{k+1}$ be a component of $G-x_{k}-x_{k}^{\prime}$ not containing $x$. Then $C_{k+1} \subseteq C_{1}$ and $C_{k+1} \cap L_{k}=\emptyset$. By applying Lemma 6 to $E_{G}\left(x_{k}, C_{k+1}\right)$ and $E_{G}\left(x_{k}^{\prime}, C_{k+1}\right)$, there exist contractible edges $x_{k} y_{k+1}$ and $x_{k}^{\prime} y_{k+1}^{\prime}$ where $y_{k+1} \in C_{k+1}$ and $y_{k+1}^{\prime} \in C_{k+1}$. Since $x \notin C_{k+1}$ and all edges in $E_{G}\left(X, X^{\prime}\right)$ are non-contractible, $y_{k+1} \in X$ and $y_{k+1}^{\prime} \in X^{\prime}$. Choose a path $Q_{k+1}$ in $C_{k+1}$ between $y_{k+1}$ and $y_{k+1}^{\prime}$. Then there exists an $X$ - $X^{\prime}$ edge $x_{k+1} x_{k+1}^{\prime}$ on $Q_{k+1}$ such that $V\left(y_{k+1} Q_{k+1} x_{k+1}\right) \subseteq X$ and $x_{k+1}^{\prime} \in X^{\prime}$. Define $P_{k+1}:=x_{k} y_{k+1} \cup y_{k+1} Q_{k+1} x_{k+1}, P_{k+1}^{\prime}:=x_{k}^{\prime} y_{k+1}^{\prime} \cup y_{k+1}^{\prime} Q_{k+1} x_{k+1}^{\prime}, R_{k+1}:=R_{k} \cup P_{k+1}$, $R_{k+1}^{\prime}:=R_{k}^{\prime} \cup P_{k+1}^{\prime}$ and $L_{k+1}:=L_{k} \cup P_{k+1} \cup P_{k+1}^{\prime} \cup x_{k+1} x_{k+1}^{\prime}$. Note that $L_{k+1} \subseteq G\left[C_{1} \cup x_{0} \cup x_{0}^{\prime}\right]$ and $G\left[C \cup L_{k+1}-x_{k+1}-x_{k+1}^{\prime}\right]$ is connected.

Define $R:=\bigcup_{k \geqslant 0} R_{k}, R^{\prime}:=\bigcup_{k \geqslant 0} R_{k}^{\prime}$ and $L:=\bigcup_{k \geqslant 0} L_{k}$. Then $L \subseteq G\left[C_{1} \cup x_{0} \cup x_{0}^{\prime}\right]$ and $x \notin \bar{L}$.

Theorem 13. Let $G$ be a 2-connected locally finite infinite graph and $G_{C}$ be the subgraph induced by all the contractible edges in $G$. Then $\overline{G_{C}}$ is 2-connected.

Proof. Suppose $\overline{G_{C}}$ is not 2-connected. Then there exists a point $x$ in $\overline{G_{C}}$ such that $\overline{G_{C}} \backslash x$ is not connected. Let $U$ and $U^{\prime}$ be two disjoint non-empty open sets in $|G|$ such that $\overline{G_{C}} \backslash x \subseteq U \cup U^{\prime},\left(\overline{G_{C}} \backslash x\right) \cap U \neq \emptyset$ and $\left(\overline{G_{C}} \backslash x\right) \cap U^{\prime} \neq \emptyset$. Define $X:=\left(\overline{G_{C}} \backslash x\right) \cap U \cap V(G)$ and $X^{\prime}:=\left(\overline{G_{C}} \backslash x\right) \cap U^{\prime} \cap V(G)$. Since $G_{C}$ is spanning, $X \cup X^{\prime}=V(G \backslash x)$. Suppose $U$ contains an interior point $a$ of an edge $b c$ of $G_{C}$. Then $\overline{G_{C}} \backslash x$ contains half edges $[b a]$ or $[c a]$ of $b c$. By the connectedness of half edge, $U$ contains $b$ or $c$. Suppose $U$ contains an end $\omega$ of $|G|$. Then $U$ contains a basic open neighborhood of $\omega$, say $\hat{C}(S, \omega)$, and thus contains infinitely many vertices. The same arguments hold for $U^{\prime}$. Therefore, both $X$ and $X^{\prime}$ are non-empty. Since $G \backslash x$ is connected, $E_{G}\left(X, X^{\prime}\right)$ is non-empty.

Suppose $x$ is a vertex or an end of $G$. Then all edges in $E_{G}\left(X, X^{\prime}\right)$ are non-contractible and $\left(X, X^{\prime}\right)$ is a partition of $V(G \backslash x)$. Suppose $x$ is an interior point of an edge $e$. Then all edges in $E_{G}\left(X, X^{\prime}\right)$ are non-contractible unless $e \in E_{G}\left(X, X^{\prime}\right) \cap E_{C}$. Note that, $E_{G}\left(X, X^{\prime}\right)-e$ is non-empty as $G$ is 2 -connected and every edge in $E_{G}\left(X, X^{\prime}\right)-e$ is noncontractible. Let $e=y y^{\prime}$ where $y \in X$ and $y^{\prime} \in X^{\prime}$. Suppose $X=\{y\}$. By Lemma 5(a), since $y$ is incident to at least two contractible edges, there is a contractible $X$ - $X^{\prime}$ edge other than $e$, which is impossible. Therefore, $|X| \geqslant 2$. Now, all edges in $E_{G}\left(X-y, X^{\prime}\right)$ are non-contractible and $\left(X-y, X^{\prime}\right)$ is a partition of $V(G \backslash y)$. In both cases, by Lemma $12, G$ contains a subdivision of a 1 -way infinite ladder $L$ such that $x \notin \bar{L}$.

Let $\omega$ be the end of $|G|$ containing $R$ and $R^{\prime}$. Note that $\omega \neq x$. Since $G_{C}$ is spanning, $\overline{G_{C}} \backslash x$ contains all the ends of $|G|$ except possibly $x$. Without loss of generality, assume $\omega \in U$. Since $U$ is open, there exists a basic open neighborhood $\hat{C}(S, \omega) \subseteq U$. Since $x_{0}^{\prime}, x_{1}^{\prime}, x_{2}^{\prime}, \ldots \in X^{\prime} \subseteq U^{\prime}$ converge to $\omega$, all but finitely many of them lie in $\hat{C}(S, \omega)$, contradicting $U \cap U^{\prime}=\emptyset$.

Finally, we prove the main result of this section, namely, $\overline{G_{C}}$ is 2 -arc-connected. This follows from a theorem by Georgakopoulos [7] concerning connected but not pathconnected subspaces of locally finite graphs. Note that since $|G|$ is Hausdorff, pathconnectedness is equivalent to arc-connectedness. 
Theorem 14 (Georgakopoulos [7]). Given any locally finite connected graph G, a connected subspace $X$ of $|G|$ is path-connected unless it satisfies the following assertions:

(1) X has uncountably many path-components each of which consists of one end only;

(2) X has infinitely many path-components that contain a vertex; and

(3) every path-component of $X$ contains an end.

Theorem 15. Let $G$ be a 2-connected locally finite infinite graph and $G_{C}$ be the subgraph induced by all the contractible edges in $G$. Then $\overline{G_{C}}$ is 2-arc-connected.

Proof. Suppose $\overline{G_{C}}$ is not 2 -arc-connected. Then there exists a point $x$ in $\overline{G_{C}}$ such that $\overline{G_{C}} \backslash x$ is not arc-connected. Note that $\overline{G_{C}} \backslash x$ is connected by Theorem 13 . By Theorem 14, $\overline{G_{C}} \backslash x$ has uncountably many path-components each of which consists of one end only. Let $\omega$ and $\omega^{\prime}$ be two such path-components of $\overline{G_{C}} \backslash x$. Since $\overline{G_{C}}$ is arc-connected by Theorem 11 , there exists an arc $A$ joining $\omega$ and $\omega^{\prime}$ in $\overline{G_{C}}$. Now, $x$ must lie in $A$ for otherwise $\omega$ and $\omega^{\prime}$ would lie in the same path-component of $\overline{G_{C}} \backslash x$. But the path-component of $\overline{G_{C}} \backslash x$ containing $\omega$ also contains $[\omega A x)$, a contradiction.

\section{Outerplanarity of 2-connected locally finite graphs}

As mentioned in the introduction, in order to extend Theorem 2 to locally finite infinite graphs, we would like to prove that for any 2-connected locally finite infinite graph $G$, if every vertex is incident to exactly two contractible edges, then $\overline{G_{C}}$ is a Hamilton circle. This requires several lemmas listed below.

Lemma 16. Let $G$ be a locally finite graph. Then every arc in $|G|$ whose two endpoints are ends contains a vertex.

Proof. Suppose $A$ is an arc in $|G|$ whose two endpoints are ends $\omega_{1}$ and $\omega_{2}$. Then there exists a finite set $S$ of vertices such that $\hat{C}\left(S, \omega_{1}\right)$ and $\hat{C}\left(S, \omega_{2}\right)$ are distinct. By the connectedness of $A, A$ contains a vertex of $S$.

Lemma 17. Let $G$ be a locally finite graph and $\omega$ be an end in $|G|$. Then every $\omega$-arc $A$ in $|G|$ contains a vertex, say $z$, and $z A$ contains a ray starting with $z$.

Proof. Denote the starting point of $A$ by $a$. First, we show that $A$ contains a vertex. If $a$ is a vertex, then we are done. If $a$ is an end, then it is true by Lemma 16. If $a$ is an interior point of an edge $x y$, then by the connectedness of $A, A$ contains $x$ or $y$.

Let $z$ be a vertex in $A$. By the connectedness of $z A, z A$ contains an interior point of an edge incident to $z$, say $z z_{1}$. Then the connectedness of $z A$ implies $z z_{1}$ lies in $z A$. Repeat the above argument for $z_{1} A$ and so on. We obtain a ray that starts with $z$ and lies in $z A$. 
Lemma 18. Let $G$ be a locally finite graph and $\omega$ be an end in $|G|$. Let $A_{1}$ and $A_{2}$ be two $\omega$-arcs in $|G|$ that are disjoint except at $\omega$. Then, for all finite subset $S$ of $V(G), \hat{C}(S, \omega)$ contains a subarc $A_{1}^{\prime} \omega A_{2}^{\prime}$ of $A_{1} \omega A_{2}$ and there is an $A_{1}^{\prime}-A_{2}^{\prime}$ path in $C(S, \omega)$.

Proof. Let $x_{1}$ be the last point of $A_{1}$ that lies in $S$ and $x_{2}$ be the last point of $A_{2}$ that lies in $S$. By Lemma 17, $x_{1} A$ contains a ray $R_{1}$ starting with $x_{1}$ and $x_{2} A$ contains a ray $R_{2}$ starting with $x_{2}$. Let $y_{1}$ be the neighbor of $x_{1}$ in $R_{1}$ and $y_{2}$ be the neighbor of $x_{2}$ in $R_{2}$. Then $y_{1} A_{1} \omega A_{2} y_{2}$ lies in $\hat{C}(S, \omega)$. Also there is a $y_{1}-y_{2}$ path in $C(S, \omega)$ which automatically contains a $y_{1} A_{1}-y_{2} A_{2}$ path.

We also need a result on the characterization of a topological circle in $|G|$ in terms of its vertex and end degrees.

Lemma 19 (Bruhn and Stein [1]). Let $C$ be a subgraph of a locally finite graph $G$. Then $\bar{C}$ is a circle if and only if $\bar{C}$ is connected and every vertex and end of $|G|$ in $\bar{C}$ has degree two in $\bar{C}$.

Now, we can proceed with the proof.

Theorem 20. Let $G$ be a 2-connected locally finite infinite graph and $G_{C}$ be the subgraph induced by all the contractible edges in $G$. If every vertex of $G$ is incident to exactly two contractible edges, then $\overline{G_{C}}$ is a Hamilton circle.

Proof. Since $G_{C}$ is spanning, $\overline{G_{C}}$ contains all vertices and ends of $|G|$. By Theorem 11, $\overline{G_{C}}$ is arc-connected. Obviously, every vertex of $G$ has degree two in $\overline{G_{C}}$. Therefore, it remains to prove that every end of $|G|$ has degree two in $\overline{G_{C}}$.

Claim 21. Let $A$ be an arc in $\overline{G_{C}}$ and $x$ be a vertex in $A$. Suppose that both $x^{-}$and $x^{+}$ exist in $A$. Let $y$ be any neighbor of $x$ other than $x^{-}$and $x^{+}$. Then every $x^{-}-x^{+}$arc in $|G|$ intersects $\{x, y\}$.

Proof. Since $x x^{-}$and $x x^{+}$are the only contractible edges incident to $x, x y$ is noncontractible. Lemma 6 implies that $G-x-y$ has exactly two components, and $x^{-}$and $x^{+}$lie in different components. By the connectedness of an arc, every $x^{-}-x^{+}$arc in $|G|$ intersects $\{x, y\}$.

Claim 22. Let $\omega$ be an end in $|G|$. Suppose $A_{1}$ and $A_{2}$ are two edge-disjoint $\omega$-arcs in $\overline{G_{C}}$. Then $A_{1}$ and $A_{2}$ can intersect only at the ends of $|G|$ with the only possible exception being that the starting points of $A_{1}$ and $A_{2}$ are the same vertex.

Proof. Obviously, $A_{1}$ and $A_{2}$ cannot intersect at an interior point of an edge. Suppose $A_{1}$ and $A_{2}$ intersect at a vertex $x$. If $x$ is not the starting point for both $A_{1}$ and $A_{2}$, then the degree of $x$ in $\overline{G_{C}}$ is at least three, a contradiction.

Claim 23. Let $\omega$ be an end in $|G|$. Suppose $A_{1}$ and $A_{2}$ are two edge-disjoint $\omega$-arcs in $\overline{G_{C}}$ such that the starting points of $A_{1}$ and $A_{2}$ are distinct vertices. Then there exists an end $\omega^{\prime}$ in $|G|$ such that there are three $\omega^{\prime}$-arcs in $\overline{G_{C}}$ that are disjoint except at $\omega^{\prime}$ unless $A_{1} \cap A_{2}=\{\omega\}$. 
Proof. Suppose $A_{1} \cap A_{2} \neq\{\omega\}$. Let $\omega^{\prime}$ be the first point of $A_{2}$ that intersects $A_{1}$. By Claim $22, \omega^{\prime}$ is an end in $|G|$ different from $\omega$. Then $A_{1} \omega^{\prime}, A_{2} \omega^{\prime}$ and $\omega A_{1} \omega^{\prime}$ are the required three $\omega^{\prime}$-arcs.

Claim 24. Let $\omega$ be an end in $|G|$. Suppose there are three edge-disjoint $\omega$-arcs in $\overline{G_{C}}$. Then there exists an end $\omega^{\prime}$ in $|G|$ such that there are three $\omega^{\prime}$-arcs in $\overline{G_{C}}$ that are disjoint except at $\omega^{\prime}$.

Proof. Let $A_{1}, A_{2}, A_{3}$ be three edge-disjoint $\omega$-arcs in $\overline{G_{C}}$. By Lemma 17 , for each $i \in$ $\{1,2,3\}, A_{i}$ contains a ray $R_{i}$. Denote the first edge of $R_{i}$ by $x_{i} y_{i}$. By Claim $22, y_{1}, y_{2}, y_{3}$ are all distinct. Therefore, without loss of generality, we can assume that the starting points of $A_{1}, A_{2}, A_{3}$ are all distinct vertices. Consider $A_{1}$ and $A_{2}$. If $A_{1} \cap A_{2} \neq\{\omega\}$, then the claim follows from Claim 23. Suppose $A_{1} \cap A_{2}=\{\omega\}$. If $A_{2} \cap A_{3} \neq\{\omega\}$, then again the claim follows from Claim 23. Therefore, suppose $A_{2} \cap A_{3}=\{\omega\}$. But then, $A_{1}, A_{2}, A_{3}$ are the desired three $\omega$-arcs.

Claim 25. For each end $\omega$ in $|G|, \operatorname{deg}_{G_{C}}(\omega) \leqslant 2$.

Proof. Suppose there are three edge-disjoint $\omega$-arcs in $\overline{G_{C}}$. By Claim 24, there exists an end $\omega^{\prime}$ in $|G|$ such that there are three $\omega^{\prime}$-arcs in $\overline{G_{C}}$ that are disjoint except at $\omega^{\prime}$. Denote these three $\omega^{\prime}$-arcs by $A_{1}, A_{2}, A_{3}$. By Lemma 16, without loss of generality, we can assume $A_{1}, A_{2}, A_{3}$ start with vertices $a_{1}, a_{2}, a_{3}$ respectively.

By applying Lemma 18 to $A_{1}$ and $A_{2}$ with $S=\left\{a_{1}, a_{2}\right\}$, we obtain an $a_{1}^{+} A_{1}-a_{2}^{+} A_{2}$ path $P$. Let $x_{1}=P \cap A_{1}, x_{2}=P \cap A_{2}$ and $x$ be the neighbor of $x_{1}$ in $Q$. If $P$ intersects $A_{3}$, then interchange $A_{2}$ and $A_{3}$. Therefore, without loss of generality, there is an $a_{1}^{+} A_{1}-a_{2}^{+} A_{2}$ path $P$ that does not intersect $A_{3}$.

Now, apply Lemma 18 to $x_{2} A_{2}$ and $A_{3}$ with $S=V(P)$. We obtain an $x_{2}^{+} A_{2}-A_{3}$ path $Q$ not intersecting $P$. Let $y_{2}=Q \cap x_{2}^{+} A_{2}$ and $y_{3}=Q \cap A_{3}$. By Claim 21, $Q$ cannot intersect $A_{2} x_{2}^{-}$, and $Q$ cannot intersect both $A_{1} x_{1}^{-}$and $x_{1}^{+} A_{1}$. Suppose $Q \cap A_{1} x_{1}^{-} \neq \emptyset$. Let $y$ be the first vertex of $Q$ that lies in $A_{1} x_{1}^{-}$. Then $x_{1}^{-} A_{1} y Q y_{2} A_{2} \omega^{\prime} A_{1} x_{1}^{+}$is an $x_{1}^{-} x_{1}^{+}$arc not intersecting $\left\{x_{1}, x\right\}$, contradicting Claim 21. Suppose $Q \cap x_{1}^{+} A_{1} \neq \emptyset$. Then there is an $x_{1}^{+} A_{1}-A_{3}$ subpath in $Q$ not intersecting $A_{2}$, and we interchange $A_{1}$ and $A_{2}$. Therefore, without loss of generality, we can assume that there is an $x_{2}^{+} A_{2}-A_{3}$ path $Q$ that does not intersect $P \cup A_{1} \cup A_{2} x_{2}^{-}$. Let $u_{2}$ be the neighbor of $y_{2}$ in $Q$ and $u_{3}$ be the neighbor of $y_{3}$ in $Q$.

Finally, apply Lemma 18 to $x_{1} A_{1}$ and $y_{2} A_{2}$ with $S=V(P \cup Q)$. We obtain an $x_{1}^{+} A_{1^{-}}$ $y_{2}^{+} A_{2}$ path $R$ not intersecting $P \cup Q$. Let $z_{1}=R \cap x_{1}^{+} A_{1}$ and $z_{2}=R \cap y_{2}^{+} A_{2}$. By Claim 21, $R$ cannot intersect $A_{1} x_{1}^{-}$and $R$ cannot intersect $A_{2} y_{2}^{-}$. Also, $R$ cannot intersect both $A_{3} y_{3}^{-}$and $y_{3}^{+} A_{3}$. Suppose $R \cap A_{3} y_{3}^{-} \neq \emptyset$. Let $z$ be the last vertex of $R$ that lies in $A_{3} y_{3}^{-}$. Then $y_{3}^{-} A_{3} z R z_{2} A_{2} \omega^{\prime} A_{3} y_{3}^{+}$is an $y_{3}^{-}-y_{3}^{+}$arc not intersecting $\left\{y_{3}, u_{3}\right\}$, contradicting Claim 21. Suppose $R \cap y_{3}^{+} A_{3} \neq \emptyset$. Let $z^{\prime}$ be the first vertex of $R$ that lies in $y_{3}^{+} A_{3}$. Then $y_{2}^{-} A_{2} x_{2} P x_{1} A_{1} z_{1} R z^{\prime} A_{3} \omega^{\prime} A_{2} y_{2}^{+}$is an $y_{2}^{-}-y_{2}^{+}$arc not intersecting $\left\{y_{2}, u_{2}\right\}$, contradicting Claim 21. Therefore, $R \cap\left(A_{1} x_{1}^{-} \cup A_{2} y_{2}^{-} \cup A_{3} \cup P \cup Q\right)=\emptyset$. But, $y_{2}^{-} A_{2} x_{2} P x_{1} A_{1} z_{1} R z_{2} A_{2} y_{2}^{+}$ is an $y_{2}^{-}-y_{2}^{+}$arc not intersecting $\left\{y_{2}, u_{2}\right\}$, contradicting Claim 21.

Claim 26. For each end $\omega$ in $|G|$, $\operatorname{deg}_{G_{C}}(\omega)=2$. 
Proof. Let $x$ be a vertex in $G_{C}$. Since $\overline{G_{C}}$ is arc-connected, there is an $\omega$-arc $A$ in $\overline{G_{C}}$ joining $x$ to $\omega$. Let $y$ be the neighbor of $x$ in $A$ and $a$ be an interior point of $x y$. Since $\overline{G_{C}}$ is 2-connected, $\overline{G_{C}} \backslash a$ is connected. Suppose $\overline{G_{C}-x y}$ is not connected. Then there exist two disjoint nonempty open sets $U$ and $V$ in $|G|$ such that $\overline{G_{C}-x y} \subseteq U \cup V$, $U \cap \overline{G_{C}-x y} \neq \emptyset$ and $V \cap \overline{G_{C}-x y} \neq \emptyset$. If $x, y \in U$, then $U \cup[x, a) \cup[y, a)$ and $V$ are two disjoint open sets in $|G|$ both intersecting $\overline{G_{C}} \backslash a$, and their union contains $\overline{G_{C}} \backslash a$, which is impossible. If $x \in U$ and $y \in V$, then $U \cup[x, a)$ and $V \cup[y, a)$ are two disjoint open sets in $|G|$ both intersecting $\overline{G_{C}} \backslash a$, and their union contains $\overline{G_{C}} \backslash a$, which is also impossible. Therefore, $\overline{G_{C}-x y}$ is connected and is arc-connected by Lemma 10. Let $A^{\prime}$ be an $x-\omega$ arc in $\overline{G_{C}-x y}$. If $y A \omega \cap A^{\prime}$ contains a vertex $u$, then $u$ has degree at least three in $G_{C}$, a contradiction. Let $\omega^{\prime}$ be the first point in $y A \omega \cap A^{\prime}$ which is an end. If $\omega^{\prime} \neq \omega$, then $\operatorname{deg}_{G_{C}}\left(\omega^{\prime}\right) \geqslant 3$ contradicting Claim 25. Therefore, $\omega^{\prime}=\omega$ and we have $\operatorname{deg}_{G_{C}}(\omega) \geqslant 2$. By Claim $25, \operatorname{deg}_{G_{C}}(\omega)=2$.

We are now ready to prove the infinite analog of Theorem 2 .

Theorem 27. Let $G$ be a 2-connected locally finite graph nonisomorphic to $K_{3}$. Then the following are equivalent:

(1) Every vertex of $G$ is incident to exactly two contractible edges.

(2) Every finite bond of $G$ contains exactly two contractible edges.

(3) $G$ is outerplanar.

Proof.

(2) $\Rightarrow(1)$ Trivial.

$(1) \Rightarrow(3)$ By Theorem 2, this is true for finite $G$. Therefore, assume $G$ is infinite. Suppose every vertex of $G$ is incident to exactly two contractible edges. By Theorem $20, \overline{G_{C}}$ is a Hamilton circle. All edges in $E(G) \backslash E_{C}$ are chords of $\overline{G_{C}}$ and are noncontractible. Consider any chord $x y$ of $\overline{G_{C}}$. Since every vertex of $G$ is incident to exactly two contractible edges, by Lemma $6, G-x-y$ consists of exactly two components $C_{1}$ and $C_{2}$. Without loss of generality, assume that $x^{+} \overline{G_{C}} y^{-} \subseteq \overline{C_{1}}$ and $y^{+} \overline{G_{C}} x^{-} \subseteq \overline{C_{2}}$. Then there is no chord of $\overline{G_{C}}$ between $x^{+} \overline{G_{C}} y^{-}$and $y^{+} \overline{G_{C}} x^{-}$. Hence, no chords of $\overline{G_{C}}$ are overlapping. Embed $\overline{G_{C}}$ in a circle of $\mathbb{R}^{2}$ and denote the embedding by $\phi$. Now, draw every chord $x y$ of $\overline{G_{C}}$ as a straight line segment between $\phi(x)$ and $\phi(y)$ in $\mathbb{R}^{2}$. This shows that $G$ is outerplanar.

$(3) \Rightarrow(2)$ Let $B$ be a finite bond of $G$ between two components $X$ and $Y$ of $G-B$. By Lemma $5(\mathrm{a}), B$ contains at least two contractible edges. Suppose $B$ contains three contractible edges $x_{1} y_{1}, x_{2} y_{2}, x_{3} y_{3}$ such that $x_{1}, x_{2}, x_{3} \in X$ and $y_{1}, y_{2}, y_{3} \in Y$. Since $X$ and $Y$ are connected, there exists a path $P$ in $X$ joining $x_{1}$ to $x_{2}$ and a path $Q$ in $Y$ joining $y_{1}$ to $y_{2}$. Let $C:=P \cup x_{1} y_{1} \cup Q \cup x_{2} y_{2}$. Obviously, $x_{3} y_{3} \notin E(C)$. Take any $x_{3}-P$ path $P^{\prime}$ in $X$ joining $x_{3}$ to $P$ at $x$ and any $y_{3}-Q$ path $Q^{\prime}$ in $Y$ joining $y_{3}$ to $Q$ at $y$. Let $R^{\prime}:=P^{\prime} \cup x_{3} y_{3} \cup Q^{\prime}$. If $R^{\prime}=x_{3} y_{3}$, then $x_{3} y_{3}$ is a chord of $C$. Since $x_{3} y_{3}$ is contractible, the two components of $C-x_{3}-y_{3}$ are joined by a path, say $R$. Then $C \cup R \cup R^{\prime}$ is a 
$K_{4}$-subdivision and $G$ is not outerplanar. Suppose $R^{\prime} \neq x_{3} y_{3}$. If both $x-y$ paths in $C$ are not edges, then $C \cup R^{\prime}$ is a $K_{2,3}$-subdivision and $G$ is not outerplanar. If one of the two $x-y$ paths in $C$ is an edge, then without loss of generality, assume $x_{2}=x$ and $y_{2}=y$. Since $x_{2} y_{2}$ is contractible, the two components of $\left(C \cup R^{\prime}\right)-x_{2}-y_{2}$ are joined by a path, say $R$. Then $C \cup R \cup R^{\prime}$ is a $K_{4}$-subdivision and $G$ is not outerplanar.

\section{Acknowledgements}

The author would like to thank Henning Bruhn for suggesting Theorem 8, and the referees for comments that greatly improve the accuracy and presentation of the paper.

\section{References}

[1] H. Bruhn and M. Stein, On end degrees and infinite circuits in locally finite graphs, Combinatorica 27 (2007), 269-291.

[2] G. Chartrand and F. Harary, Planar permutation graphs, Ann. Inst. H. Poincare Sect. B 3 (1967), 433-438.

[3] R. Diestel, Graph theory (4th edition), Springer-Verlag, 2010.

[4] R. Diestel and D. Kühn, On Infinite Cycles I, Combinatorica 24 (2004), 69-89.

[5] R. Diestel and D. Kühn, On Infinite Cycles II, Combinatorica 24 (2004), 91-116.

[6] R. Diestel and D. Kühn, Topological paths, cycles and spanning trees in infinite graphs, Eur. J. Comb. 25 (2004), 835-862.

[7] A. Georgakopoulos, Connected but not path-connected subspaces of infinite graphs, Combinatorica 27 (2007), 683-698.

[8] M. Kriesell, A survey on contractible edges in graphs of a prescribed vertex connectivity, Graphs Comb. 18 (2002), 1-30.

[9] M. Kriesell, Average degree and contractibility, J. Graph Theory 51 (2006), 205-224.

[10] W. Mader, Generalizations of critical connectivity of graphs, Discrete Math. 72 (1988), 267-283.

[11] W.T. Tutte, A theory of 3-connected graphs, Nederl. Akad. Wet., Proc., Ser A 64 (1961), 441-455.

[12] H. Wu, Distribution of contractible elements in 2-connected matroids, Discrete Math. 305 (2005), 386-393. 